\title{
Acute GvHD prophylaxis with posttransplant cyclophosphamide after hematopoietic stem cell transplantation (HSCT) for non-malignant disorders
}

Tatiana A. Bykova, Anastasia S. Borovkova, Anna A. Osipova, Varvara N. Ovechkina, Olesya V. Paina, Polina V. Kozhokar, Alexander L. Alyanskyi, Alexander D. Kulagin, Elena V. Semenova, ${ }^{\star B}$ Boris I. Smirnov, Ludmila S. Zubarovskaya, Boris V. Afanasyev

R. Gorbacheva Memorial Research Institute of Children Oncology, Hematology and Transplantology; Department of Hematology, Transfusiology and Transplantology, The First St. Petersburg State I. Pavlov Medical University, St. Petersburg, Russia

*The St. Petersburg State Electrotechnical University (LETI), St. Petersburg, Russia

Dr. Tatiana A. Bykova, R. Gorbacheva Memorial Research Institute of Children Oncology, Hematology and Transplantology, The First St. Petersburg State I. Pavlov Medical University, L. Tolstoy St 6-8, 197022, St. Petersburg, Russian Federation

\section{Summary}

Transplantation of allogeneic hematopoietic stem cells (allo-HSCT) is an effective treatment method for non-malignant diseases and inherited disorders. Development of acute graft-versus-host-disease (aGVHD) is a negative factor with adverse effects upon clinical outcomes. Usage of "novel" schedules for drug prophylaxis of this complication using posttransplant cyclophosphamide (PtCy) seems to decrease the GVHD risk.

The aim of this study was to assess efficiency of PtCy as a tool for aGVHD prevention in the patients with non-malignant diseases of hematopoiesis and inherited syndromes.

\section{PATIENTS AND METHODS}

97 patients with non-malignant blood disorders and metabolic diseases underwent allo-HSCT at the R. Gorbacheva Memorial Institute of Children Oncology and Transplantation over a period of 2005 to 2018. A total of 118 HSCTs were carried out. The aGVHD prophylaxis in 89 cases was performed by a standard schedule (with calcineurin inhibitors). 29 patients were treated according to PtCy regimen, at a dose of $50 \mathrm{mg} / \mathrm{kg}$ at days +3 and +4 .
Phone: +7(905) 2076776

E-mail: dr.bykova@mail.ru

\section{RESULTS}

Cumulative frequency of acute GVHD comprised 32\%. Patients treated with PtCy exhibited lower rates of this condition compared to the group with standard prophylaxis schedule (26\% vs 47\%, p=0.05). Frequency of skin aGVHD was also less common in the PtCy group ( $23 \%$ vs $45 \%, \mathrm{p}=0.046$ ); gastrointestinal aGVHD was observed at equal rates in the both groups. Stem cell engraftment after nonmyeloablative conditioning in HSCT patients with subsequent PtCy administration proved to be sufficiently weaker compared to other patients (86 vs $50 \%, \mathrm{p}=0.004)$. In conclusion, posttransplant GVHD prevention based on cyclophosphamide prophylaxis is an efficient method which may decrease aGVHD risk. However, one should take into account a higher non-engraftment rate as a potential hazard of HSCT when using non-myeloablative conditioning regimens and PtCy-based GVHD prophylaxis.

\section{Keywords}

Allogeneic hematopoietic stem cell transplantation, non-malignant disorders, acute graft-versus-host disease, cyclophosphamide prophylaxis. 


\section{Introduction}

Allogeneic transplantation of hematopoietic stem cells (allo-HSCT) is considered an integral component of most treatment protocols aimed for therapy of hematological malignancies and solid tumors as well as some genetic diseases in children and adolescents. It is a method of choice for the patients with non-malignant clinical conditions intended for correction of inherited deficiency typical to the given syndrome, repopulation of the immune system by normal cells, or replenishment of a deficient enzyme, e.g., in storage diseases $[1,2]$. Choosing an optimally compatible donor is a key factor determining favorable outcome in HSCT [3]. An HLA-compatible unrelated donor is not available for ca. $15-20 \%$ of the patients, because of extreme allelic variability of HLA system. Lower HLA compatibility is associated with additional risks of severe posttransplant immune complications, e.g., graft-versus-host disease. Pharmacological prevention of acute GVHD is based on combined usage of different medications, i.e., calcineurin inhibitors, cytostatic drugs (metothrexate, micophenolate mophetyl), m-TOR inhibitors, antithymocyte immunoglobulins. Cyclophosphamide at early terms post-transplant (days $+3+4)$ is considered as a novel approach to aGvHD prophylaxis after HSCT (PTCy). The mean purpose of this therapy is to abrogate effects of activated alloreactive T lymphocytes, thus allowing to decrease acute GVHD risk by $30 \%$. However, most published data describes treatment of adult patients with hematological malignancies $[5,6]$, several studies in pediatric HSCT are also based on this category of patients. Hence, the aim of the present study was to assess efficiency of PTCy therapy in pediatric patients with non-malignant diseases.

\section{Patients and Methods}

Over the time period of 2005 to March 2018, we observed ninety-seven patients with various non-malignant diseases subjected to allo-HSCT at the clinic of R. Gorbacheva Memorial Institute of Children Oncology, Hematology and Transplantation. A total of 118 allo-HSCT were performed including 21 cases (18\%) of repeated transplants, due to initial graft failure, or secondary rejection. The primary non-malignant conditions were represented by the following disorders: hemoglobinopathies, 8 patients $(8 \%)$; bone marrow insufficiency (both inborn and acquired), 44 cases (46\%); metabolic diseases, 35 cases (36\%), primary immune deficiencies, 10 patients (10\%).

Acute GVHD (aGVHD) prophylaxis in majority of HSCT cases was based on calcineurin inhibitors $(n=89,75 \%)$. Posttransplant cyclophosphamide (PtCy) was administered in 29 cases (25\%), at the dose of $50 \mathrm{mg} / \mathrm{kg}$ weight (days +3 and +4 after HSCT). This schedule of GVHD prophylaxis was most often in type 1 mucopolysaccharidosis (Hurler syndrome) $(n=9)$, beta-thalassemia $(n=9)$. In 11 cases $(38 \%)$, HSCT was performed from haploidentical donors, or as a repeated transplant $(n=9,31 \%)$. Myeloablative and reduced-intensity conditioning regimens were applied at similar rates (respectively, for 15 and 14 cases).

\section{Results}

The two-year survival rates in total group did not substantially differ between standard GVHD prophylaxis schedule, and the PtCy protocol (62\% versus 64\%) (Fig. 1A). A number of factors did sufficiently improve this parameter: patient's age (under 5 years old) by the moment of HSCT (77\% vs $50 \%, \mathrm{p}=0.004$, see Fig. 1B); shorter time period (under 2 years) from diagnosis to allo-HSCT (74\% vs 47\%, $\mathrm{p}=0.003$, see Fig. 1C), transplant engraftment ( $72 \%$ vs $44 \%, \mathrm{p}=0.001$, see Fig. 1D).

Successful engraftment was documented in 91 cases. Cumulative engraftment rates did not differ between the groups with standard protocol and PtCy prophylaxis (70\% vs $84 \%$, see Fig. 2A). Likewise, we have not revealed any significant differences for the groups treated according to MAC and RIC schedules ( $87.5 \%$ vs $77 \%$, $\mathrm{p}=0.31$, see Fig. $2 \mathrm{~B}$ ). However, the patients subjected to non-myeloablative conditioning followed by Cy treatment showed a definitely lower engraftment rate ( 86 vs 50\%, p=0.004, see Fig. 2C).

Stem cell engraftment in our patients was dependent on the donor type. I.e., the patients who underwent HSCT from HLA-compatible donor (either related or unrelated) showed higher engraftment frequency than the patients who have got stem cells from haploidentical donor (92\% vs $84 \%$ vs $58 \%, \mathrm{p}=0.05$, see Fig. 3).

The primary disease for which allo-HSCT was performed was also of importance. E.g., the patients with primary immune deficiencies demonstrated engraftment in all cases. The lowest engraftment rate was observed in patients with hemoglobinopathies. Functioning graft among the patients who received second HSCT due to failure of the first transplant, was achieved in only $46 \%$ of cases.

Cumulative incidence (CI) of aGVHD rate in post-HSCT patients was $32 \%$ of total. The patients with PtCy had lower CI aGVHD if compared to the group with standard prophylaxis ( $26 \%$ vs $47 \%, \mathrm{p}=0.05$, Fig. $4 \mathrm{~A})$. CI of aGVHD with skin affection was also significantly lower in the PtCy group (23\% vs $45 \%, \mathrm{p}=0.046)$ as seen from the Fig. 4B. Intestinal and hepatic aGVHD occurred in the both groups at comparable rates. The inter-group distribution for severity grade was also similar.

Clinical results of PtCy treatment were specially evaluated for the most homogenous group of the patients with Hurler syndrome (type $1 \mathrm{MPS})$. This cohort was represented by 22 allo-HSCT, with PtCy prophylaxis in six cases. Overall survival was similar for the patients subjected to different aGVHD prophylaxis (82\% at standard aGVHD prophylaxis versus $100 \%$ in PtCy group, see Fig. 5A). Clinical engraftment was achieved in all cases, whereas CI of aGVHD was $63 \%$ in the standard prophylaxis group against $34 \%$ for the PtCy group (Fig. 5B). Frequency of life-threatening GVHD (stage III to IV) did not differ significantly (20\% versus $18 \%$, Fig. 5C). 
A
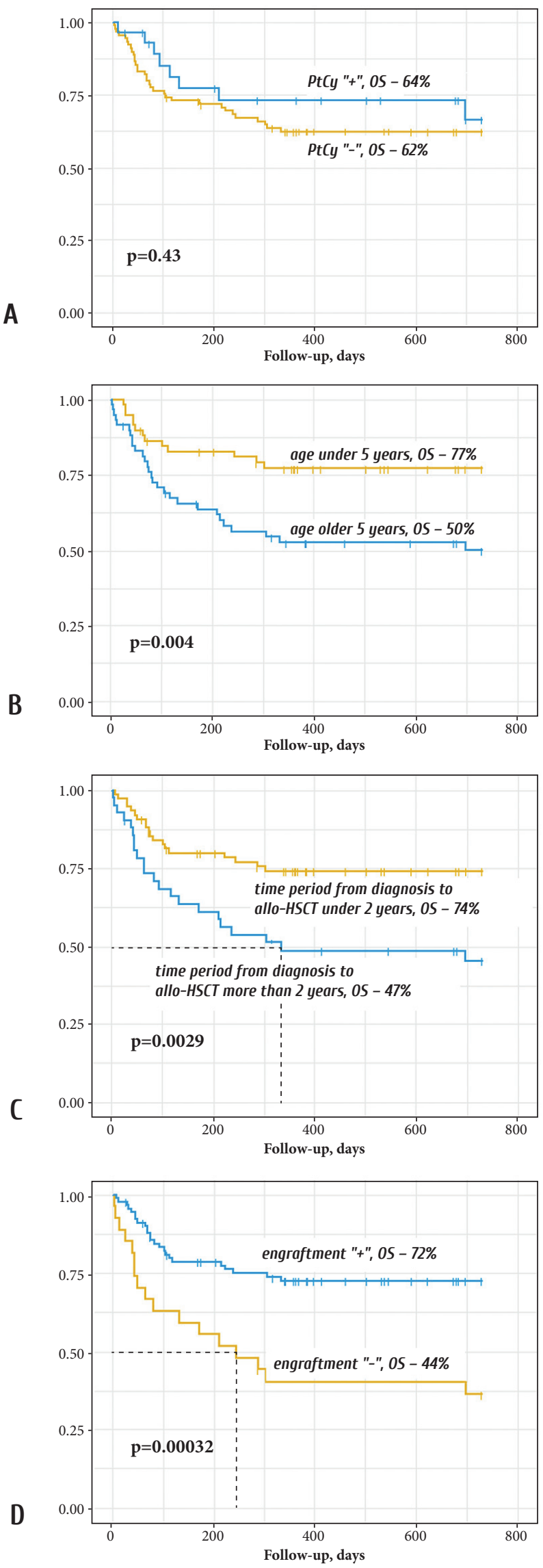

Figure 1. (A) Overall 2-year survival of the patients when using standard GHVD prophylaxis, and PtCy treatment; (B) Overall 2-year survival dependent on the age at HSCT; (C) Dependence on time period between primary diagnosis and allo-HSCT; (D) Transplant engrafted. Abscissa, terms after HSCT, days; ordinate, survival rates
A
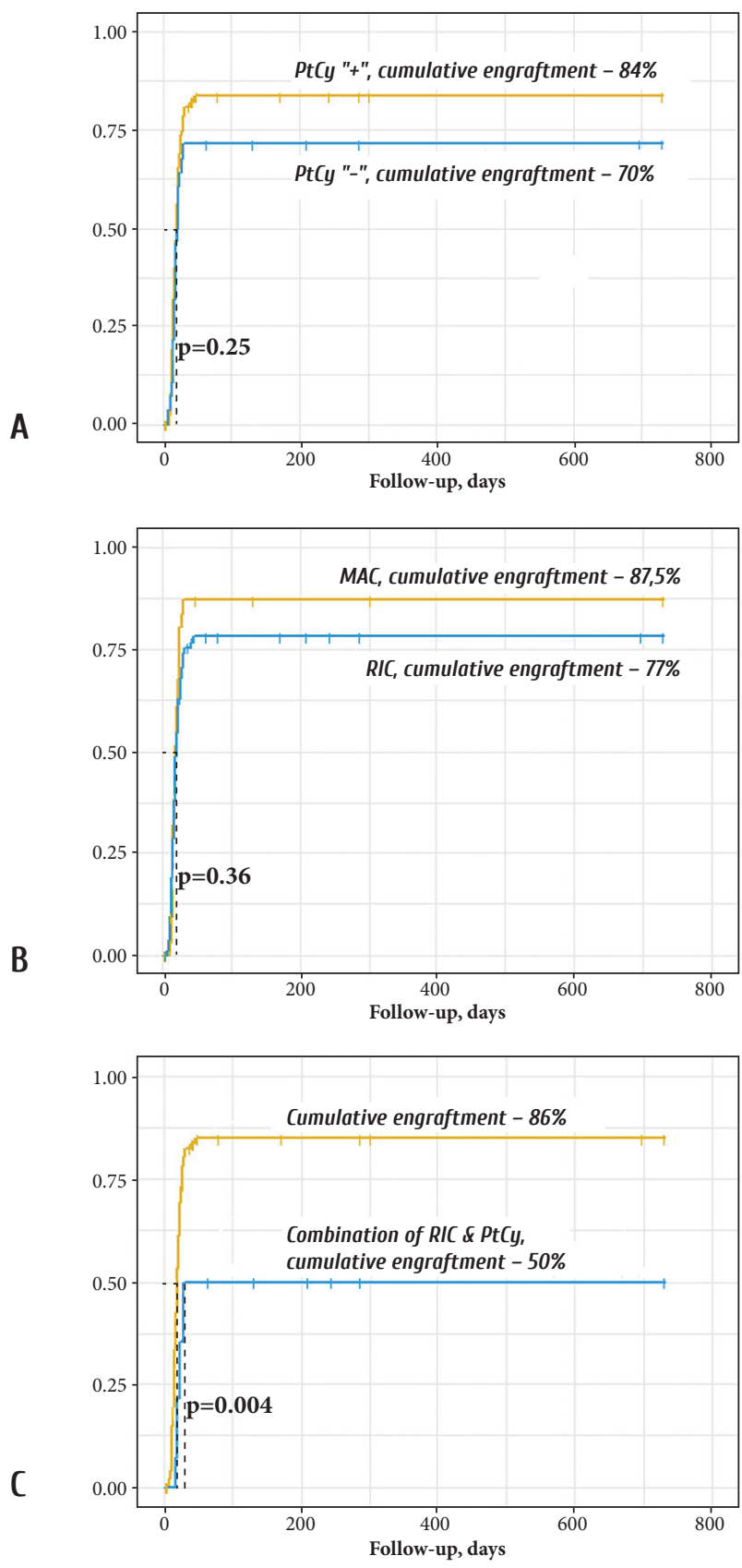

Figure 2. (A) Cumulative engraftment rates in patients at a standard GVHD prophylaxis versus $\mathrm{PtCy}$ protocol; (B) dependence on the conditioning intensity; (C) dependence on the combination of conditioning regimen and prophylaxis. Abscissa, terms after HSCT, days; ordinate, aGVHD frequency 
Table 1. Primary clinical conditions in the patients with non-malignant disorders

\begin{tabular}{|c|c|}
\hline Types of the disorders & $\mathbf{N}$ \\
\hline \multicolumn{2}{|c|}{ Hemoglobinopathies } \\
\hline Beta-thalassemia major & 8 \\
\hline \multicolumn{2}{|c|}{ Bone marrow failure syndromes } \\
\hline Idiopathic aplastic anemia & 27 \\
\hline Fanconi anemia & 10 \\
\hline Kostmann syndrome & 3 \\
\hline Blackfan-Diamond anemia & 3 \\
\hline Shwachman-Diamond anemia & 1 \\
\hline \multicolumn{2}{|c|}{ Metabolic diseases } \\
\hline Mucopolysaccharidosis type 1, Hurler syndrome & 19 \\
\hline Autosomal recessive osteopetrosis & 7 \\
\hline X-linked adrenoleukodystrophy & 4 \\
\hline Globoid cell leukodystrophy & 3 \\
\hline Metachromatic leidystrophy & 1 \\
\hline Farber disease & 1 \\
\hline \multicolumn{2}{|c|}{ Primary immune deficiencies } \\
\hline Wiskott Aldrich syndrome & 7 \\
\hline Severe combined immune deficiency (SCID) & 2 \\
\hline Chediak-Higashi syndrome & 1 \\
\hline
\end{tabular}

Table 2. Demographic and clinical characteristics of the patients subjected to acute GVHD prophylaxis based on calcineurin inhibitors versus post-transplant cyclophosphamide (Cy)

\begin{tabular}{|c|c|c|c|}
\hline Parameter & $\begin{array}{l}\text { Standard aGVHD } \\
\text { prophylaxis }\end{array}$ & $\begin{array}{l}\text { Post-transplant Cy-based } \\
\text { prophylaxis }\end{array}$ & $p$ \\
\hline Number of patients & 89 & 29 & \\
\hline Age, years & 6 years (9 mo to 21 years) & 3.6 years (9 mo to 27 years) & \\
\hline $\begin{array}{l}\text { Gender } \\
\text { Male } \\
\text { Female }\end{array}$ & $\begin{array}{l}39 \\
50\end{array}$ & $\begin{array}{l}19 \\
10\end{array}$ & 0.05 \\
\hline $\begin{array}{l}\text { Diagnosis: } \\
\text { Hemoglobinopathies } \\
\text { Bone marrow failure syndromes, including: } \\
\text { Aplastic anemias } \\
\text { Metabolic diseases, i.e., } \\
\text { Mucopolysaccharidosis type 1, Hurler syndrome } \\
\text { Primary immune deficiencies } \\
\end{array}$ & $\begin{array}{l}4 \\
48 \\
31 \\
30 \\
16 \\
7 \\
\end{array}$ & $\begin{array}{l}6 \\
8 \\
5 \\
11 \\
6 \\
4 \\
\end{array}$ & 0.06 \\
\hline $\begin{array}{l}\text { Allo-HSCT number } \\
\text { First HSCT } \\
\text { Repeated HSCT }\end{array}$ & $\begin{array}{l}77 \\
12\end{array}$ & $\begin{array}{l}20 \\
9\end{array}$ & 0.03 \\
\hline $\begin{array}{l}\text { Donor type: } \\
\text { Allogeneic unrelated } \\
\text { Allogeneic related } \\
\text { Haploidentical }\end{array}$ & $\begin{array}{l}67 \\
15 \\
7\end{array}$ & $\begin{array}{l}13 \\
4 \\
11\end{array}$ & 0.02 \\
\hline $\begin{array}{l}\text { Conditioning regimen } \\
\text { Myeloablative } \\
\text { Reduced-intensity conditioning }\end{array}$ & $\begin{array}{l}18 \\
72\end{array}$ & $\begin{array}{l}15 \\
13\end{array}$ & 0.00 \\
\hline $\begin{array}{l}\text { Source of graft } \\
\text { Bone marrow (BM) } \\
\text { Peripheral blood stem cells (PBSC) } \\
\text { Combined BM+PBSC+umbilical stem cells }\end{array}$ & $\begin{array}{l}54 \\
32 \\
3\end{array}$ & $\begin{array}{l}15 \\
9 \\
3\end{array}$ & \\
\hline
\end{tabular}


Table 3. aGVHD frequency and distribution by severity for the groups with standard (calcineurin inhibitor-based) prophylaxis, and PtCy-administration

\begin{tabular}{|c|c|c|c|}
\hline & Standard aGVHD prophylaxis & PtCy-based aGVHD prophylaxis & $\mathrm{p}$ \\
\hline \multicolumn{4}{|c|}{ Cumulative incidence, $\%$} \\
\hline acute GVHD, number of cases & 47 & 26 & 0.05 \\
\hline \multicolumn{4}{|c|}{ Distribution by severity grades } \\
\hline $\begin{array}{l}1^{\text {st }} \\
2^{\text {nd }} \\
3^{\text {rd }} \\
4^{\text {th }} \\
\end{array}$ & $\begin{array}{l}10(26 \%) \\
13(33 \%) \\
5(13 \%) \\
11(28 \%) \\
\end{array}$ & $\begin{array}{l}1(12,5 \%) \\
2(25 \%) \\
1(12,5 \%) \\
4(50 \%) \\
\end{array}$ & \\
\hline \multicolumn{4}{|c|}{ Cumulative incidence, $\%$} \\
\hline Skin aGVHD & 45 & 23 & 0,046 \\
\hline \multicolumn{4}{|c|}{ Distribution by severity grade } \\
\hline $\begin{array}{l}1^{\text {st }} \\
2^{\text {nd }} \\
3^{\text {rd }} \\
4^{\text {th }}\end{array}$ & $\begin{array}{l}12(33 \%) \\
9(24 \%) \\
13(35 \%) \\
3(8 \%) \\
\end{array}$ & $\begin{array}{l}2(33 \%) \\
3(50 \%) \\
1(17 \%) \\
\end{array}$ & \\
\hline \multicolumn{4}{|c|}{ Cumulative incidence, $\%$} \\
\hline acute gut GVHD & 25 & 23 & ns \\
\hline \multicolumn{4}{|c|}{ Distribution by severity grade } \\
\hline $\begin{array}{l}1^{\text {st }} \\
2^{\text {nd }} \\
3^{\text {dd }} \\
4^{\text {th }}\end{array}$ & $\begin{array}{l}4(19 \%) \\
6(29 \%) \\
4(19 \%) \\
7(33 \%)\end{array}$ & $\begin{array}{l}1(17 \%) \\
2(33 \%) \\
3(50 \%)\end{array}$ & \\
\hline \multicolumn{4}{|c|}{ Cumulative incidence frequency, \% } \\
\hline Hepatic aGVHD & 7 & 7 & ns \\
\hline \multicolumn{4}{|c|}{ Distribution by severity grade } \\
\hline $\begin{array}{l}1^{\text {st }} \\
2^{\text {nd }}\end{array}$ & $\begin{array}{l}2(33 \%) \\
4(67 \%)\end{array}$ & $\begin{array}{l}1(50 \%) \\
1(50 \%)\end{array}$ & \\
\hline
\end{tabular}

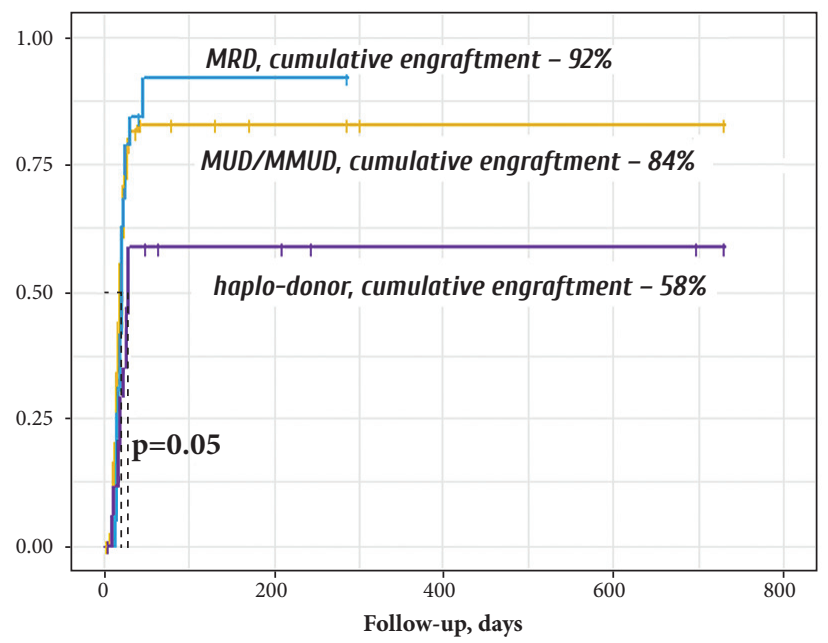

Figure 3. Cumulative engraftment rate (ordinate) in the patients is dependent on the donor type 


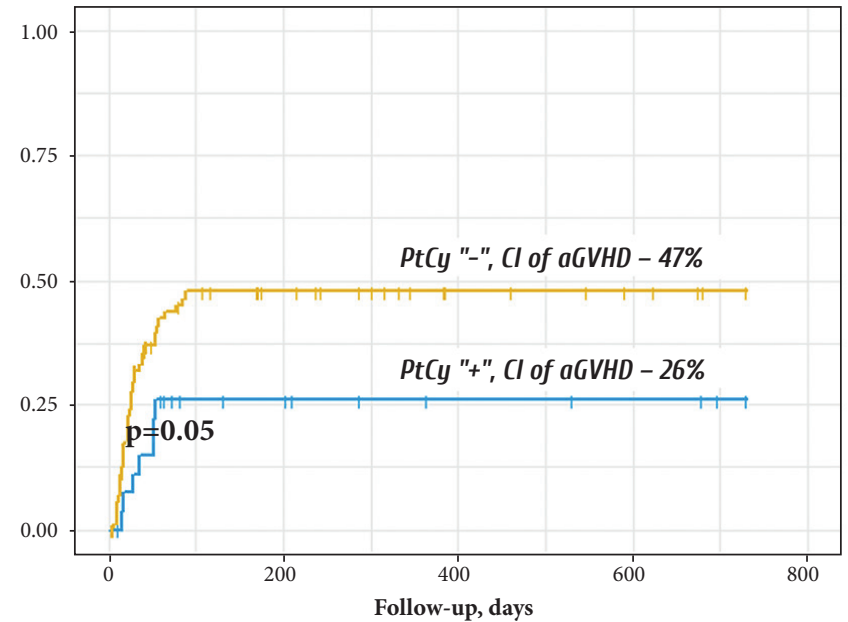

A

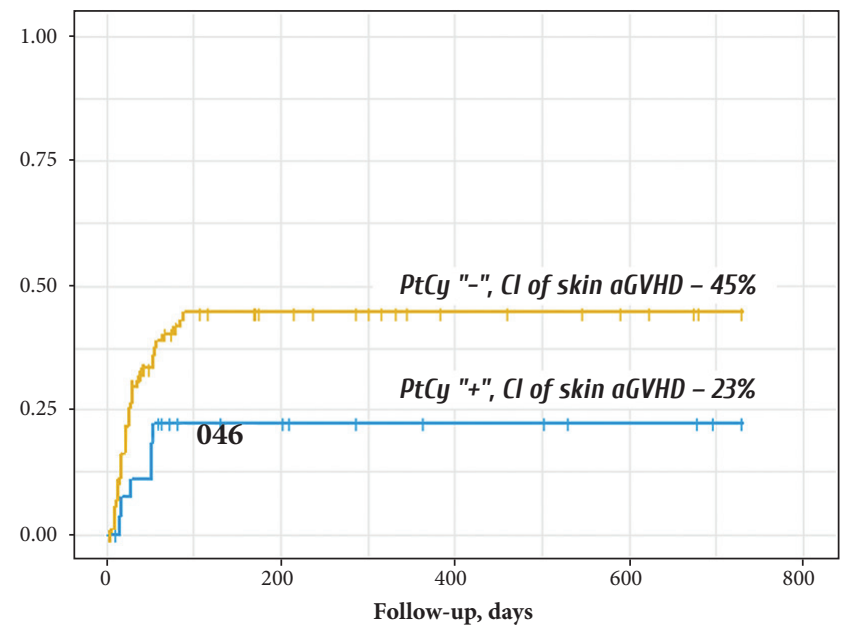

B

Figure 4. (A) Total Cl of aGVHD (ordinate); (B) Cl of skin aGVHD among the patients subjected to standard GVHD prophylaxis and PtCy treatment. Abscissa, Cl of aGVHD; ordinate, terms after HSCT, days

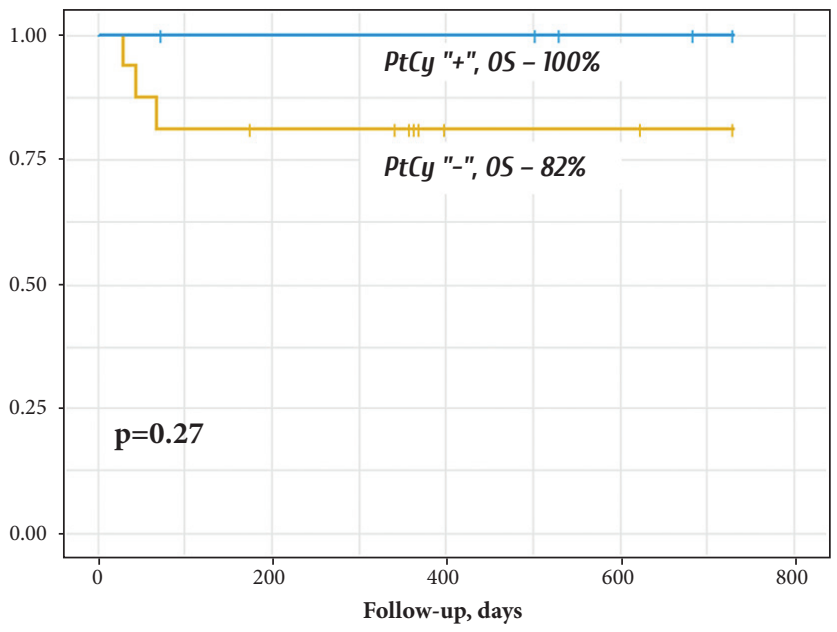

A

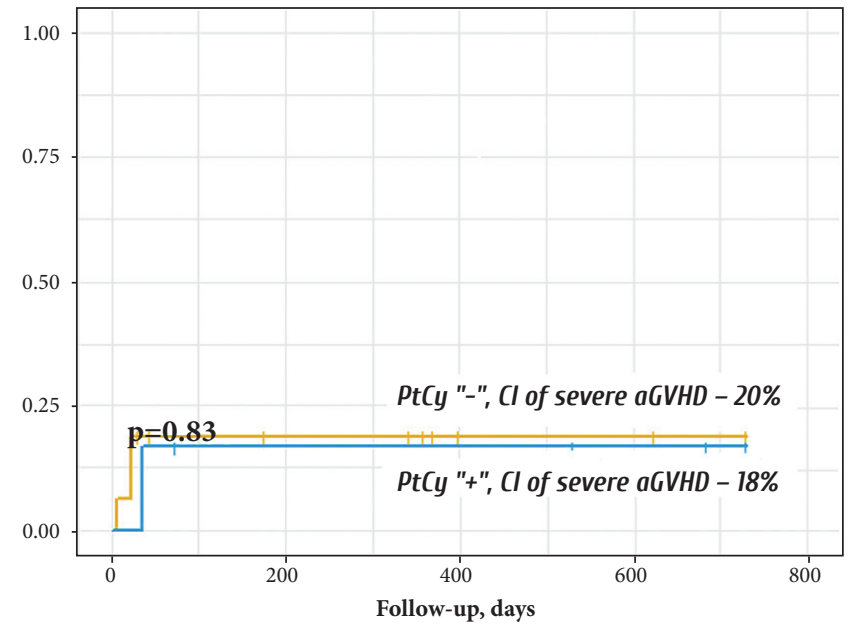

C

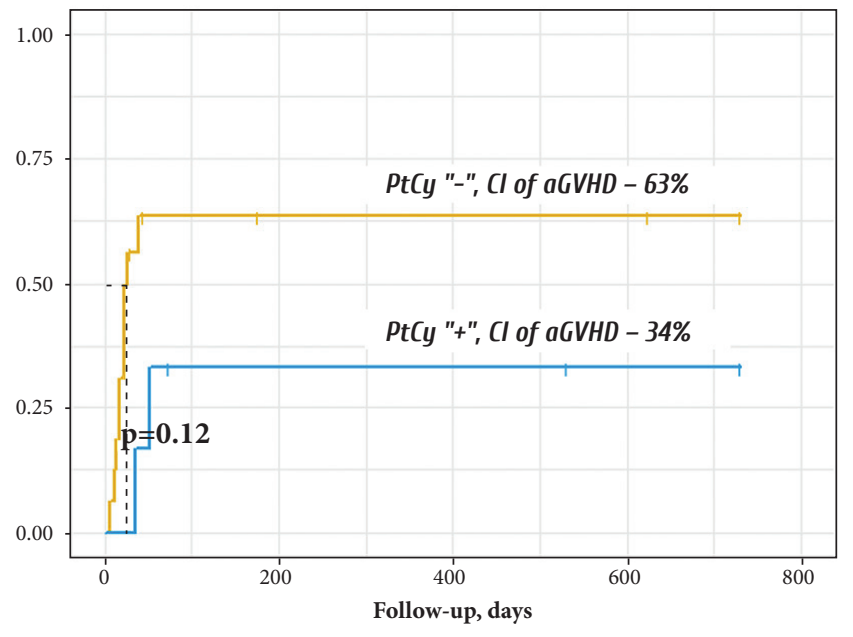

B

Figure 5. (A) Overall survival among patients with Hurler syndrome (type 1 MPS), dependent on different GVHD prophylaxis schedules; (B) $\mathrm{Cl}$ of aGVHD frequency; (C) Severe aGVHD (grade III to IV) in the patients undergoing standard GVHD prophylaxis, or PtCy protocol. Abscissa, GVHD frequency; ordinate, terms after HSCT, days 


\section{Discussion}

Search for a fully HLA-matched donor for HSCT is critical to the patients with non-malignant diseases. Due to ethnic background of the patients with thalassemia, autosomal recessive osteopetrosis etc., they are unlikely to find a compatible donor. Time is also an important factor, especially for the patients with primary immune deficiencies or storage metabolic diseases which extends the prospective for recruitment of alternative stem cell donors [7]. Allo-HSCT from a nonrelated donor or partially compatible haploidentical donor exhibit comparable survival parameters for the patients with non-malignant disorders. Under these conditions, the PtCy-based GHD prophylaxis provides good control of evolving aGVHD [8]. A higher risk of non-engraftment in cases of haploidentical donorship could be decreased due to myeloablative conditioning regimens. The recruitment of haploidentical donors for HSCT in children with primary immune deficiencies and sickle-cell anemia have been described in present studies $[9,10,11]$. PtCy prophylaxis was applied in all these cases showing its clinical efficiency. This approach has additional benefits when applying peripheral blood stem cells as a source of transplant $[8,12]$.

\section{Conclusion}

aGVHD prevention based on cyclophosphamide prophylaxis is an effective treatment which may decrease risk of aGVHD specially in skin affection when compared to standard treatment methods based on calcineurin inhibitors. However, higher non-engraftment rate can be a potential hazard of HSCT performed in patients with non-malignant disorders when using non-myeloablative conditioning regimens and PtCy-based GVHD prophylaxis.

\section{Conflict of interest}

No conflicts of interest are reported.

\section{References}

1. Passweg JR, Baldomero H, Bader P, Bonini C, Cesaro S, Dreger P, Duarte RF, Dufour C, Falkenburg JH, Farge-Bancel D, Gennery A, Kröger N, Lanza F, Nagler A, Sureda A, Mohty M; European Society for Blood and Marrow Transplantation (EBMT). Hematopoietic SCT in Europe 2013: recent trends in the use of alternative donors showing more haploidentical donors but fewer cord blood transplants. Bone Marrow Transplant 2015;50(4):476-482.

2. Afanasyev BV, Zubarovskaya LS, Moiseev IS. Allogeneic hematopoietic stem cell transplantation in children: current issues and prospectives. Russian J Pediatric Hematol Oncol. 2015; 2(2): 28-42 (In Russian).

3. Afanasyev BV, Zubarovskaya LS, Alyansky AL. Paina OV, Borovkova AS, Kuzmich EV, Bykova TA, Deev RV, Isaev AA. Donor selection in allogeneic hematopoietic stem cell transplantation. Russian J Pediatric Hematol Oncol. 2016; 3(3): 30-36 (In Russian).
4. Owens AH, Santos GW. The effect of cytotoxic drugs on graft-versus-host disease in mice. Transplantation. 1971. 4(11): 378-382.

5. Moiseev IS, Pirogova OV, Babenko EV, Gindina TL, Darskaya EI, Morozova EV, Bondarenko SN, Afanasyev BV. Single-agent post-transplantation cyclophosphamide versus calcineurin-based graft-versus-host disease prophylaxis in matched related bone marrow transplantation. Cell Ther Transplant. 2017; 6(4): 52-59.

6. Moiseev IS, Pirogova OV, Alyanski AL, Babenko EV, Gindina TL, Darskaya EI, Slesarchuk OA, Bondarenko SN, Afanasyev BV. Graft-versus-host disease prophylaxis in unrelated peripheral blood stem cell transplantation with post-transplantation cyclophosphamide, tacrolimus, and mycophenolate mofetil. Biol Blood Marrow Transplant. 2016; 22(6):1037-1042.

7. Booth C, Silva J, Veys P. Stem cell transplantation for the treatment of immunodeficiency in children: current status and hopes for the future. Expert Rev Clin Immunol. 2016;12(7):713-723.

8. Jaiswal SR, Chakrabarti A, Chatterjee S, Ray K, Chakrabarti S. Haploidentical transplantation in children with unmanipulated peripheral blood stem cell graft: The need to look beyond post-transplantation cyclophosphamide in younger children. Pediatr Transplant. 2016;20(5):675-82.

9. Rastogi N, Katewa S, Thakkar D, Kohli S, Nivargi S, Yadav SP. Reduced-toxicity alternate-donor stem cell transplantation with posttransplant cyclophosphamide for primary immunodeficiency disorders. Pediat Blood Cancer. 2018;65(1). doi: $10.1002 / p b c .26783$.

10. Thakkar D, Katewa S, Rastogi N, Kohli S, Nivargi S, Yadav SP. Successful reduced intensity conditioning alternate donor stem cell transplant for Wiskott-Aldrich syndrome. J Pediat Hematol Oncol. 2017;39(8):e493-e496.

11. Wiebking V, Hütker S, Schmid I, Immler S, Feuchtinger T, Albert MH. Reduced toxicity, myeloablative HLA-haploidentical hematopoietic stem cell transplantation with post-transplantation cyclophosphamide for sickle cell disease. Ann Hematol. 2017;96(8):1373-1377.

12. Jaiswal SR, Chakrabarti A, Chatterjee S, Ray K, Chakrabarti S. Haploidentical transplantation in children with unmanipulated peripheral blood stem cell graft: The need to look beyond post-transplantation cyclophosphamide in younger children. Pediatr Transplant.2016;20(5):675-682. 


\title{
Профилактика острой реакции «трасплантат против хозяина» с применением циклофосфамида после трансплантации гемопоэтических стволовых клеток пациентам с неопухолевыми заболеваниями
}

\author{
Татьяна А. Быкова, Анастасия С. Боровкова, Анна А. Осипова, Варвара Н. Овечкина, Олеся В. Паина, \\ Полина В. Кожокарь, Александр Л. Алянский, Александр Д. Кулагин, Елена В. Семенова, `Борис И. Смирнов, \\ Людмила С. Зубаровская, Борис В. Афанасьев
}

Научно-исследовательский институт детской онкологии, гематологии и трансплантологии имени Р. М. Горбачевой и кафедра гематологии, трансфузиологии и трансплантологии, Федеральное государственное бюджетное образовательное учреждение высшего образования «Первый Санкт-Петербургский государственный медицинский университет имени академика И. П. Павлова» Министерства здравоохранения Российской Федерации

*Санкт-Петербургский государственный электротехнический университет «ЛЭТИ», Санкт-Петербург, Россия

\section{Резюме}

Аллогенная трансплантация гемопоэтических стволовых клеток - эффективный метод терапии незлокачественных заболеваний системы кроветворения и наследственных синдромов. Фактором, значимо влияющим на ухудшение прогноза, является развитие острой реакции «трансплантат против хозяина» (оРТПХ). Использование «новых» схем фармакологической профилактики данного осложнения на основе посттрансплантационного циклофосфамида (ПТЦ) позволяет снизить вероятность его развития.

\section{ЦЕЛЬ РАБОТЫ}

Оценить эффективность использования ПТЦ в качестве профилактики оРТПХ у пациентов с незлокачественными заболеваниями системы кроветворения и наследственными синдромами.

\section{ПАЦИЕНТЫ И МЕТОДЫ}

В клинике НИИ ДОГиТ им. Р. М. Горбачевой наблюдается 97 пациентов с различными незлокачественными заболеваниями системы кроветворения и наследственными синдромами, которым в период с 2005 по март 2018 года выполнено 118 алло-ТГСК. В качестве профилактики оРТПХ у 89 пациентов использовались схемы на основе ингибиторов кальциневрина, в 29 случаях на основе ПТЦ в дозе 50 мг/кг на Д+3, Д+4.

\section{РЕЗУЛЬТАТЫ}

Кумулятивная частота развития оРТПХ составила $32 \%$. Пациенты с использованием ПТЦ имели ниже уровень данного осложнения в сравнении с группой стандартной профилактики (26\% vs 47\%, p=0,05), также кумулятивная частота оРТПХ с поражением кожи была значимо ниже в группе с ПТЦ (23\% vs $45 \%, \mathrm{p}=0,046)$, частота развития оРТПХ с поражением желудочно-кишечного тракта, печени были сопоставимы в обеих группах. Показатель приживления трансплантата у пациентов, получивших немиелоаблативные режимы с последующим введением ПЦТ был значимо ниже в сравнении с остальной группой (86 vs 50\%p=0,004).

\section{ЗАКЛЮЧЕНИЕ}

Профилактика оРТПХ на основе Посттрансплантационного циклофосфамида является эффективным методом, снижающим вероятность развития оРТПХ. Однако, у пациентов с незлокачественными заболеваниями необходимо учитывать факт возможного увеличения частоты неприживления трансплантата при использовании немиелоаблативных режимов кондиционирования и профилактики на основе ПТЦ.

\section{Ключевые слова}

Аллогенная трансплантация гемопоэтических стволовых клеток, неопухолевые заболевания, острая реакция «трансплантат против хозяина», профилактика циклофосфамидом. 